\title{
Improved Limits on Cosmogenic Fluxes from Ultra-High Energy Cosmic Rays
}

\section{Kathrine Mørch Groth, ${ }^{*}$ Yoann Genolini and Markus Ahlers}

Niels Bohr Institute, University of Copenhagen, DK-2100 Copenhagen, Denmark

E-mail: xsq168@alumni.ku.dk

Ultra-high energy cosmic rays (UHE CRs) interacting with the cosmic radiation background produce two cosmogenic messengers: neutrinos with energies in the $\mathrm{EeV}$ range and gamma rays accumulating in the $\mathrm{GeV}-\mathrm{TeV}$ range. The most optimistic scenario for cosmogenic fluxes assumes the dominance of protons above the Greisen-Zatsepin-Kuzmin threshold of resonant scattering with photons in the cosmic microwave background. Whereas these optimistic cosmogenic fluxes are testable with present observatories, the corresponding predictions of heavier UHE CR composition models are orders of magnitude smaller, falling within the domain of more sensitive future detectors. In this study we use the latest results of the Pierre Auger observatory for the UHE CR spectrum and chemical composition to derive conservative lower limits on the cosmogenic neutrino and gamma ray fluxes. We investigate the prospects and requirements of future large-scale neutrino and CR observatories to observe these fluxes.

$37^{\text {th }}$ International Cosmic Ray Conference (ICRC 2021)

July 12th - 23rd, 2021

Online - Berlin, Germany

\footnotetext{
${ }^{*}$ Presenter
} 


\section{Introduction}

The propagation of ultra-high energy (UHE) cosmic rays (CRs) is affected by their interactions with cosmic radiation backgrounds. Above an energy of $E_{\mathrm{GZK}} \simeq 50 \mathrm{EeV} \mathrm{CR}$ protons scatter resonantly with photons of the cosmic microwave background (CMB) via the $\Delta(1232)$ resonance whereas CR nuclei are photo-dissociated via the giant dipole resonance (GDR). This limits the propagation distance to about $100-200 \mathrm{Mpc}[1,2]$. The observed spectrum above $E>E_{\mathrm{GZK}}$ is therefore expected to receive contributions only from local cosmic ray sources.

Pion production via photo-hadronic processes above $E_{\mathrm{GZK}}$ is visible in the form of cosmogenic neutrinos and $\gamma$-rays in the EeV energy range [3]. These fluxes are guaranteed contributions to the cosmic neutrino and $\gamma$-ray backgrounds that follow directly from the observation of UHE CRs and the existence of cosmic photon backgrounds. In particular, cosmogenic neutrinos are one of the main targets of next-generation neutrino observatories that are sensitive in the EeV energy range. The detection (or upper limits) of these neutrinos can provide valuable information about the composition of UHE CRs and hence their possible sources.

Cosmogenic flux predictions in the EeV range have a strong dependence on the mass composition of UHE CRs above $E_{\mathrm{GZK}}$. To estimate the required flux sensitivity of future neutrino observatories, it is of general interest to derive a minimal contribution consistent with the observed UHE CR spectra and composition. We follow here the idea of Ref. [4] that lower limits on cosmogenic fluxes can be estimated from the observed composition of UHE CRs, rather than the initial chemical composition at the source - information that gets rapidly washed out by GDR cascades.

In these proceedings, we will reevaluate the results of Ref. [4] using recent observations of the Pierre Auger observatory and state-of-the-art CR propagation models. We will start in section 2 with a summary of cosmic ray propagation effects and emission of cosmogenic contributions. We will then give an estimate of cosmogenic neutrino and $\gamma$-ray spectra in section 3 that are based on the observed UHE CR spectrum and the average observed mass composition above $E_{\mathrm{GZK}}$. We then conclude in section 4 .

\section{Propagation of UHE CR Nuclei}

Over sufficiently large distances, the distribution of UHE CR sources can be treated as continuous and homogeneous. In this case, the evolution of the flux of UHE CR nuclei is governed by a set of (Boltzmann) continuity equations of the form

$$
\dot{Y}_{i}=\partial_{E}\left(H E Y_{i}\right)+\partial_{E}\left(b_{i} Y_{i}\right)-\Gamma_{i}^{\mathrm{tot}} Y_{i}+\sum_{j} \int \mathrm{d} E_{j} \gamma_{j i} Y_{j}+\mathcal{L}_{i}
$$

where $Y_{i}$ is the comoving number density of particle type $i$ related to the physical density $n_{i}$ as $Y_{i}=(1+z)^{-3} n_{i}$. We assume the standard flat $\Lambda$ CDM universe with Hubble rate $H^{2}=H_{0}^{2}\left(\Omega_{\Lambda}+\right.$ $\left.\Omega_{M}(1+z)^{3}\right)$ with $\Omega_{M}=0.31, \Omega_{\Lambda}=0.69$ and $H_{0} \simeq 67 \mathrm{~km} \mathrm{~s}^{-1} \mathrm{Mpc}^{-1}$ [5]. Redshift and coordinate time are related as $\dot{z}=-(1+z) H$. The first two terms on the r.h.s. of Eq. (1) describe energy loss via cosmic expansion and other continuous energy losses defined via $b_{i}=-\mathrm{d} E / \mathrm{d} t$. The third and forth term describe particle losses via inelastic interactions with total rates $\Gamma_{i}^{\text {tot }}$ and generations $j \rightarrow i$ via differential interaction rates $\gamma_{j i}$. The last term $\mathcal{L}_{i}$ denotes the comoving emission rate 
density of particle type $i$. The detailed derivation of the interaction rates and energy losses from a given photon background is discussed, e.g. in Refs. [6, 7].

The relevant interactions for UHE CR nuclei during propagation are interactions with the cosmic radiation backgrounds. Besides the CMB there are also infra-red to ultra-violet contributions at higher energies [8,9] as well as radio backgrounds at lower energies $[10,11]$ from the emission of stars and (active) galaxies. At energies of the CR ankle the dominant interaction is continuous energy loss via Bethe-Heitler pair production [12] in the CMB. This process is a coherent interaction of the UHE CR nucleons. Compared to the proton case with energy loss $b_{p}(z, E)$, the loss of heavy nuclei with mass number $A$ and charge $Z$ scales as $b_{A}(E) \simeq Z^{2} b_{p}(E / A)$.

At higher energies the propagation of UHE CR nuclei is dominated by photo-disintegration [13, 14]. Rapid interactions of UHE CR nuclei with CMB photons via the giant dipole resonance (GDR) lead to the nucleon losses such as $A \rightarrow(A-1)+N$ or $A \rightarrow(A-2)+2 N$ where $N$ indicates the disintegrated nucleons. The resonance lies at about $20 \mathrm{MeV}$ in the rest frame of the nucleus, corresponding to a CR energy of $E \simeq 2 \times 10^{10} / \epsilon_{\mathrm{meV}} \mathrm{GeV}$, where $\epsilon_{\mathrm{meV}}$ is the background photon energy in units of meV.

At the highest energies, when the photon wavelength becomes smaller than the size of the nucleus, the photon interacts with substructures like quasi-deuterons. This channel forms a plateau above about $30 \mathrm{MeV}$ in the nucleus rest frame that extends up to the pion production threshold at about $145 \mathrm{MeV}$ [15]. Resonant pion production of UHE CR protons, in particular via $\Delta(1232)$, as well as the GDR of UHE CR nuclei with CMB photons both peak at energies of a few $10^{10} \mathrm{GeV}$. Hence, independent of the chemical composition of UHE CRs we expect a cutoff (or break) in the flux of UHE CRs around $E_{\mathrm{GZK}} \simeq 50 \mathrm{EeV}$ as first pointed out by Greisen, Zatsepin and Kuzmin (GZK) [1, 2].

Photo-hadronic interactions above the pion production threshold are the dominant channels for the production of cosmogenic neutrinos and $\gamma$-rays [3]. The rapidly increasing rates at energies around $5 \times 10^{10} \mathrm{GeV}$ are due to resonances with $\mathrm{CMB}$ photons, in particular $p+\gamma \rightarrow \Delta(1232) \rightarrow$ $n+\pi^{+}$. At higher energies multi-pion production becomes relevant which forms a plateau in the cross section. In the case of UHE CR nuclei we can approximate the energy loss from photonucleon interactions above pion production threshold as $b_{A, \gamma \pi}(E) \simeq A b_{p, \gamma \pi}(E / A)$. However, see the discussion in Ref. [16].

Pions produced in photo-hadronic interactions decay via $\pi^{+} \rightarrow \mu^{+}+v_{\mu}$ and $\mu^{+} \rightarrow e^{+}+v_{e}+\bar{v}_{\mu}$ and the charged conjugate processes. The total flux of neutrinos depends on the source spectrum, composition and evolution. In general, lighter compositions and larger maximal energies with hard spectra $(\gamma \simeq 2)$ predict higher cosmogenic neutrino fluxes since the pion production threshold scales with atomic mass number. Since the UHE CR spectrum at the highest energies can only receive contributions from local sources $(r \lesssim 200 \mathrm{Mpc})$ a strong redshift evolution of the sources with an increased contribution to neutrinos is also feasible.

Cosmogenic neutrinos and $\gamma$-rays have been studied by various authors for the case of pureproton models [3, 17-23] and also mixed composition models including heavy nuclei [24-34]. In these proceedings we follow the ansatz of Ref. [4] and derive lower limits on the cosmogenic neutrino contributions which are based on the observed composition of UHE CRs, rather than the inferred composition at the sources. 

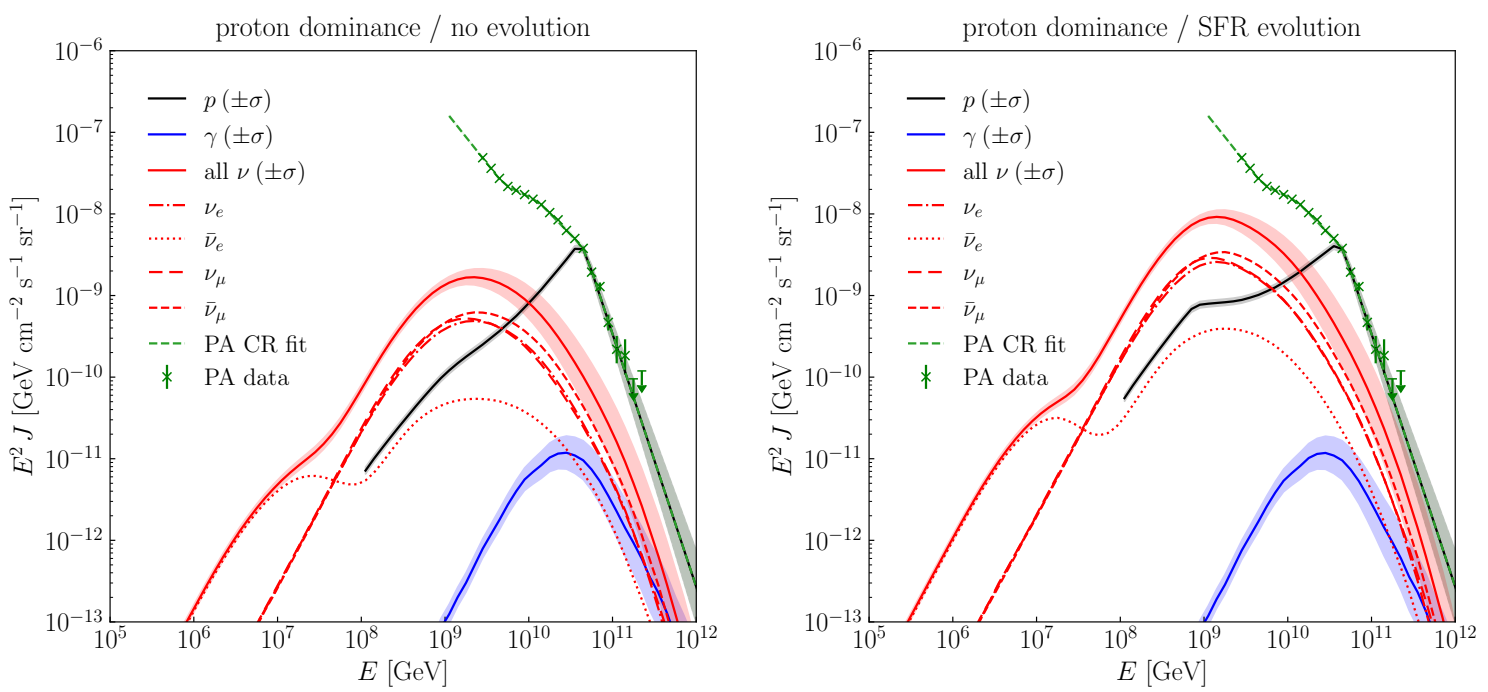

Figure 1: Cosmogenic neutrino and $\gamma$-ray fluxes from UHE CR above $50 \mathrm{EeV}$ assuming proton dominance. The proton flux (black line) is fixed to the best-fit spectrum from Pierre Auger [35, 36] (dashed green line). We show results for a constant comoving number density of sources ("no evolution"; left plot) and comoving number density following the star formation rate ("SFR evolution"; right plot). The $\gamma$-ray flux (blue lines) are attenuated by pair production in the $\mathrm{CMB}$. We also show the contribution of individual neutrino flavors on production.

\section{Minimal Cosmogenic Contributions}

The fluxes of cosmogenic neutrinos and $\gamma$-rays in the EeV energy range are produced by photohadronic interactions of cosmic ray nuclei with energies $E_{\mathrm{CR}} \gtrsim A \times 50 \mathrm{EeV}$. To first order, the cross section of this process can be approximated as the incoherent superposition of subprocesses involving the scattering of $A$ nucleons with energy $E_{\mathrm{CR}} / A$. The only competing process at these high energies are photo-disintegration of heavy nuclei. The latter process (approximately) conserves the Lorentz boost of secondary nuclei and hence the energy per nucleon.

These two arguments show that the production of cosmogenic fluxes in the $\mathrm{EeV}$ range is determined by the UHE CR nucleon spectrum above the nucleon energy $E_{\mathrm{N}} \gtrsim 50 \mathrm{EeV}$. This flux can be estimated from recent observations on the Pierre Auger Observatory [35]. The cosmic ray spectrum at these energies can be approximated by a soft power-law, $J\left(E_{\mathrm{CR}}\right) \propto E_{\mathrm{CR}}^{-\gamma}$ with $\gamma \simeq 5.2 \pm 0.4$. We will assume in the following that the CR flux above this energy is dominated by the observed mass group $A_{0}$. The nucleon spectrum above $50 \mathrm{EeV}$ is therefore approximately

$$
J_{\mathrm{N}}\left(E_{\mathrm{N}}\right) \simeq A_{\mathrm{o}}^{2} J_{\mathrm{CR}}\left(A_{\mathrm{o}} E_{\mathrm{N}}\right) \simeq A_{\mathrm{o}}^{2-\gamma} J_{\mathrm{CR}}(50 \mathrm{EeV})\left(\frac{E_{\mathrm{N}}}{50 \mathrm{EeV}}\right)^{-\gamma}
$$

with $50 \mathrm{EeV} \times J_{\mathrm{CR}}(50 \mathrm{EeV}) \simeq 0.018 \mathrm{~km}^{-2} \mathrm{sr}^{-1} \mathrm{yr}^{-1}$. In the following we will derive cosmogenic fluxes assuming proton dominance above $50 \mathrm{EeV}$. Other mass compositions follow the same energy spectra as the protons rescaled by the factor $A_{\mathrm{o}}^{2-\gamma}$. For instance, for helium we expect only about $1.2 \%$ of the cosmogenic neutrino flux with respect to the proton case.

For our fit of the UHE CR nucleon spectrum we approximate the source term in Eq. (1) in the form $\mathcal{L}_{\mathrm{N}}\left(z, E_{\mathrm{N}}\right)=\mathcal{H}(z) Q_{\mathrm{N}}\left(E_{\mathrm{N}}\right)$, where $\mathcal{H}$ accounts for the redshift evolution of comoving 

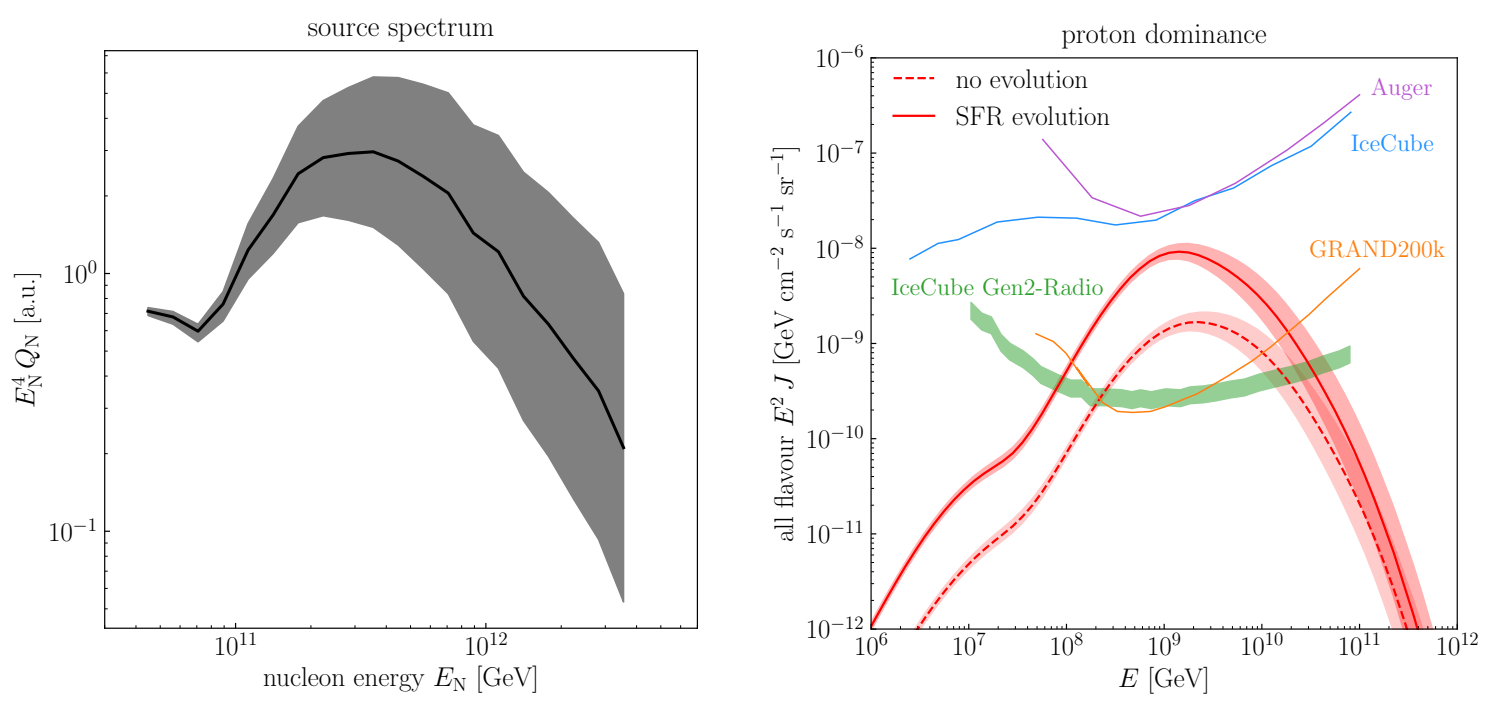

Figure 2: Left panel: Reconstructed source spectrum of UHE CR nucleons (arbitrary units). The source spectrum is practically identical for the two source evolution cases shown in Fig. 1 and follow a soft spectrum in the vicinity of $E^{-4}$. Right panel: Summary of cosmogenic neutrino fluxes assuming proton dominance of UHE CR protons above $50 \mathrm{EeV}$. We compare the expected fluxes for constant comoving source number density ("no evolution"; dashed red line) and source density evolution following the star formation rate ("SFR evolution"; solid red line). These flux predictions are consistent with limits from IceCube [37] and and Pierre Auger [38] and are in reach of future observatories, such as GRAND200k [39] and IceCube-Gen2 [40].

source density and $Q_{\mathrm{N}}(E)$ denotes the nucleon spectral emission rate from an individual source. We consider two source evolution scenarios in the following: $i$ ) The case "no evolution" considers a constant comoving number density of UHE CR sources, $\mathcal{H}(z)=\mathcal{H}(0)$, in the redshift range $0 \leq z \leq 4$. This is our most pessimistic scenario in terms of the production of cosmogenic neutrinos and $\gamma$-rays. ii) The case "SFR evolution" is a less conservative choice where the source evolution follows the star-formation rate (SFR) $\mathcal{H}_{\mathrm{SFR}}(z) \propto(1+z)^{n_{i}}$ with $n_{1}=3.4$ for $z<1$ and $n_{2}=-0.3$ for $1<z<4[41,42]$.

We use the Monte-Carlo code CRPROPA [43] to determine the secondary emission of UHE CR sources. Figure 1 shows the resulting fit to the UHE CR spectrum above $50 \mathrm{EeV}$ provided by Pierre Auger [35, 36]. The calculation assumes proton dominance in the observed spectrum, $A_{\mathrm{o}}=1$, at these energies. Note that the $\mathrm{CR}$ emission spectrum above $50 \mathrm{EeV}$ produces a tail of $\mathrm{CR}$ nucleons in the observed spectrum that extends below this energy threshold. These CR nucleons are related to secondary cosmic rays from photo-hadronic processes in the CMB. The left panel of Fig. 2 also shows our best-fit spectrum of CR nuclei above $50 \mathrm{EeV}$. The emission is soft with a spectral index that follows approximately $\gamma \simeq 4$.

Figure 1 shows also the corresponding flux of cosmogenic neutrinos and $\gamma$ rays as red and blue bands, respectively, that accounted for the statistical uncertainty of the power-law fit [35, 36] (green dashed lines). While pion production produces neutrinos and $\gamma$-rays with comparable intensity, the observable $\gamma$-ray fluxes are reduced by $e^{+} e^{-}$pair production in the CMB. Source evolution of UHE CR sources following the SFR (left panel) provides cosmogenic contributions that are a factor 5 larger than those for a constant comoving source density (right panel). 
Note that extending the UHE CR emission spectrum below $50 \mathrm{EeV}$ will necessarily increase the contribution of cosmogenic neutrinos and $\gamma$-rays. In this sense, our flux predictions can be considered as lower limits of the cosmogenic emission, that only depend on the observed average mass composition $A_{\mathrm{o}}$ above $50 \mathrm{EeV}$. The right panel of Fig. 2 shows the summary of our "minimial" cosmogenic neutrino fluxes for the two source evolution scenarios in comparison to neutrino upper limits from IceCube [37] and Pierre Auger [38] as well as the sensitivity of future neutrino observatories IceCube-Gen2 [40] and GRAND200k [39]. One can notice that future neutrino observatories are capable of detecting cosmogenic neutrino emission in the $\mathrm{EeV}$ range if the contribution of protons above $50 \mathrm{EeV}$ is at least $10 \%$. This is consistent with earlier results $[4,44]$.

As mentioned earlier, our "minimal" flux predictions have a simple scaling with observed CR mass number as $J \propto A_{\mathrm{o}}^{2-\gamma}$. For helium dominance, $A_{\mathrm{o}}=4$, this corresponds to a flux reduction of $1.2 \%$ compared to the case of proton dominance. This low flux level is below the sensitivity of nextgeneration neutrino telescope. However, our calculation does not account for cosmogenic neutrino production by the extra-galactic background light (EBL), which produces additional contribution in the sub-EeV energy range. These contributions become increasingly important for the case of heavy nuclei [4].

\section{Conclusion}

We have discussed the production of cosmogenic neutrinos and $\gamma$-rays by UHE CRs above energies of $50 \mathrm{EeV}$. At these high energies, the neutrino production can be simply estimated by the UHE CR nucleon spectrum, which can be estimated directly by the observed mass composition at Earth. We have shown that proton dominance above $50 \mathrm{EeV}$ will result in cosmogenic neutrino fluxes that are in reach of next-generation neutrino observatories.

Depending on source evolution, a contribution of $2 \%$ ("SFR evolution") or $10 \%$ ("no evolution") would lead to detectable fluxes. On the other hand, dominance of heavier nuclei above $50 \mathrm{EeV}$ will degrade the cosmogenic flux predictions as $J \propto A_{\mathrm{o}}^{2-\gamma}$, making the observation of $\mathrm{EeV}$ cosmogenic neutrinos less likely.

Cosmogenic $\gamma$-rays can also be tested by UHE CR observatories by limits on the photon fractions. The $\gamma$-ray fluxes in these proceedings have been approximated by the absorption of $\gamma$ rays via pair production with the $\mathrm{CMB}$. We are presently investigating the effect of cosmic radiation background, including the most recent estimates of the EBL and radio backgrounds as well as secondary emission of electromagnetic cascades on our flux predictions.

\section{Acknowledgements}

The authors acknowledge support from VILLUM FondEN under project no. 18994.

\section{References}

[1] K. Greisen, Phys. Rev. Lett. 16 (1966) 748-750.

[2] G. T. Zatsepin and V. A. Kuzmin, JETP Lett. 4 (1966) 78-80. 
[3] V. S. Berezinsky and G. T. Zatsepin, Yad. Fiz. 11 (1970) 200-205.

[4] M. Ahlers and F. Halzen, Phys. Rev. D 86 (2012) 083010, arXiv: 1208.4181.

[5] P. A. Zyla et al., (Particle Data Group Collaboration), PTEP 2020 no. 8, (2020) 083C01.

[6] M. Ahlers, L. A. Anchordoqui, and S. Sarkar, Phys. Rev. D 79 (2009) 083009, arXiv:0902.3993.

[7] M. Ahlers and A. M. Taylor, Phys. Rev. D 82 (2010) 123005, arXiv: 1010 . 3019.

[8] A. Franceschini, G. Rodighiero, and M. Vaccari, Astron. Astrophys. 487 (2008) 837, arXiv:0805. 1841.

[9] A. Franceschini and G. Rodighiero, Astron. Astrophys. 603 (2017) A34, arXiv: 1705.10256.

[10] R. J. Protheroe and P. L. Biermann, Astropart. Phys. 6 (1996) 45-54, arXiv:astro-ph/9605119. [Erratum: Astropart.Phys. 7, 181 (1997)].

[11] I. C. Niţu, H. T. J. Bevins, J. D. Bray, and A. M. M. Scaife, Astropart. Phys. 126 (2021) 102532, arXiv:2004.13596.

[12] G. R. Blumenthal, Phys. Rev. D 1 (1970) 1596-1602.

[13] F. W. Stecker, Phys. Rev. 180 (1969) 1264-1266.

[14] J. L. Puget, F. W. Stecker, and J. H. Bredekamp, Astrophys. J. 205 (1976) 638-654.

[15] A. Mücke, R. Engel, J. P. Rachen, R. J. Protheroe, and T. Stanev, Comput. Phys. Commun. 124 (2000) 290-314, arXiv: astro-ph/9903478.

[16] L. Morejon, A. Fedynitch, D. Boncioli, D. Biehl, and W. Winter, JCAP 11 (2019) 007, arXiv: 1904.07999.

[17] S. Yoshida and M. Teshima, Prog. Theor. Phys. 89 (1993) 833-845.

[18] C. T. Hill and D. N. Schramm, Phys. Lett. B 131 (1983) 247.

[19] R. J. Protheroe and P. A. Johnson, Astropart. Phys. 4 (1996) 253, arXiv:astro-ph/9506119.

[20] R. Engel, D. Seckel, and T. Stanev, Phys. Rev. D 64 (2001) 093010, arXiv: astro-ph/0101216.

[21] M. Ahlers, L. A. Anchordoqui, M. C. Gonzalez-Garcia, F. Halzen, and S. Sarkar, Astropart. Phys. 34 (2010) 106-115, arXiv: 1005.2620.

[22] G. B. Gelmini, O. Kalashev, and D. V. Semikoz, JCAP 01 (2012) 044, arXiv: 1107.1672.

[23] J. Heinze, D. Boncioli, M. Bustamante, and W. Winter, Astrophys. J. 825 no. 2, (2016) 122, arXiv: 1512.05988.

[24] D. Hooper, A. Taylor, and S. Sarkar, Astropart. Phys. 23 (2005) 11-17, arXiv: astro-ph/0407618.

[25] M. Ave, N. Busca, A. V. Olinto, A. A. Watson, and T. Yamamoto, Astropart. Phys. 23 (2005) 19-29, arXiv: astro-ph/0409316.

[26] D. Allard, M. Ave, N. Busca, M. A. Malkan, A. V. Olinto, E. Parizot, F. W. Stecker, and T. Yamamoto, JCAP 09 (2006) 005, arXiv : astro-ph/0605327.

[27] L. A. Anchordoqui, H. Goldberg, D. Hooper, S. Sarkar, and A. M. Taylor, Phys. Rev. D 76 (2007) 123008, arXiv:0709.0734.

[28] K. Kotera, D. Allard, and A. V. Olinto, JCAP 10 (2010) 013, arXiv: 1009.1382.

[29] S. Yoshida and A. Ishihara, Phys. Rev. D 85 (2012) 063002, arXiv: 1202.3522.

[30] A. Romero-Wolf and M. Ave, JCAP 07 (2018) 025, arXiv: 1712 . 07290. 
[31] R. Alves Batista, R. M. de Almeida, B. Lago, and K. Kotera, JCAP 01 (2019) 002, arXiv: 1806.10879.

[32] K. Møller, P. B. Denton, and I. Tamborra, JCAP 05 (2019) 047, arXiv: 1809.04866.

[33] A. van Vliet, R. Alves Batista, and J. R. Hörandel, Phys. Rev. D 100 no. 2, (2019) 021302, arXiv: 1901.01899.

[34] J. Heinze, A. Fedynitch, D. Boncioli, and W. Winter, Astrophys. J. 873 no. 1, (2019) 88, arXiv: 1901.03338.

[35] A. Aab et al., (Pierre Auger Collaboration), Phys. Rev. Lett. 125 no. 12, (2020) 121106, arXiv:2008.06488.

[36] A. Aab et al., (Pierre Auger Collaboration), Phys. Rev. D 102 no. 6, (2020) 062005, arXiv:2008.06486.

[37] M. G. Aartsen et al., (IceCube Collaboration), Phys. Rev. D 98 no. 6, (2018) 062003 , arXiv: 1807.01820.

[38] A. Aab et al., (Pierre Auger Collaboration), JCAP 10 (2019) 022, arXiv: 1906.07422.

[39] J. Álvarez-Muñiz et al., (GRAND Collaboration), Sci. China Phys. Mech. Astron. 63 no. 1, (2020) 219501, arXiv: 1810.09994.

[40] M. G. Aartsen et al., (IceCube-Gen2 Collaboration), J. Phys. G 48 no. 6, (2021) 060501, arXiv:2008.04323.

[41] A. M. Hopkins and J. F. Beacom, Astrophys. J. 651 (2006) 142-154, arXiv: astro-ph/0601463.

[42] H. Yuksel, M. D. Kistler, J. F. Beacom, and A. M. Hopkins, Astrophys. J. Lett. 683 (2008) L5-L8, arXiv: 0804. 4008.

[43] R. Alves Batista, A. Dundovic, M. Erdmann, K.-H. Kampert, D. Kuempel, G. Müller, G. Sigl, A. van Vliet, D. Walz, and T. Winchen, JCAP 05 (2016) 038, arXiv: 1603.07142.

[44] A. van Vliet, R. Alves Batista, and J. R. Hörandel, PoS ICRC2019 (2021) 1025, arXiv: 1909.01932. 